\title{
INTEGRATION OF VECTOR-VALUED PSEUDO-ALMOST PERIODIC FUNCTIONS
}

\author{
CHUANYI ZHANG
}

(Communicated by J. Marshall Ash)

\begin{abstract}
A necessary and sufficient condition is given to show that the indefinite integral of a vector-valued pseudo-almost periodic function is again pseudo-almost periodic. Then we use this result to answer a question about weakly almost periodic functions.
\end{abstract}

Throughout this paper, $X$ denotes a Banach space and $\mathbb{J}_{a}$ stands for $[a, \infty)$ when $a \in \mathbb{R}$ and for $\mathbb{R}$ when $a=-\infty ; \mathscr{C}\left(\mathbb{J}_{a}, X\right)$ denotes the space of all bounded continuous functions from $\mathbb{J}_{a}$ to $X$. Also, $m$ denotes Lebesgue measure on $\mathbb{R}$.

Let $f \in \mathscr{C}(\mathbb{R}, X)$. The translate of $f$ by $s \in \mathbb{R}$ is the function $R_{s} f(t)=$ $f(t+s), t \in \mathbb{R} . \quad f$ is called (weakly) almost periodic if the set $\left\{R_{\mathbf{R}} f\right\}=$ $\left\{R_{s} f: s \in \mathbb{R}\right\}$ is (weakly) relatively compact in $\mathscr{C}(\mathbb{R}, X)$ [3]. Denote by $(\mathscr{W} \mathscr{A} \mathscr{P}(\mathbb{R}, X)) \mathscr{A} \mathscr{P}(\mathbb{R}, X)$ all such functions.

In case $X=\mathbb{C}$, we will omit $X$ from our notation and write, for example, $\mathscr{C}\left(\mathbb{J}_{a}\right)$ for $\mathscr{C}\left(\mathbb{J}_{a}, X\right)$.

For a function $f \in \mathscr{A} \mathscr{P}(\mathbb{R})$, the classical Bohl-Bohr integration theorem (cf. [2]) asserts that the indefinite integral $F(t)=\int_{0}^{t} f(u) d u$ will also be almost periodic on $\mathbb{R}$ whenever $F$ is bounded. For $f \in \mathscr{A} \mathscr{P}(\mathbb{R}, X)$, there is Kadets's generalized Bohl-Bohr theorem (see [4]). A question arises naturally in $\mathscr{W} \mathscr{A} \mathscr{P}(\mathbb{R}, X)$; that is, if $f \in \mathscr{W} \mathscr{A} \mathscr{P}(\mathbb{R}, X)$, what is a necessary and sufficient condition for $F$ to be again in $\mathscr{W} \mathscr{A} \mathscr{P}(\mathbb{R}, X)$ ?

Let $f \in \mathscr{W} \mathscr{A} \mathscr{P}(\mathbb{R}, X)$. Then $f=g+\varphi$, where $g \in \mathscr{A} \mathscr{P}(\mathbb{R}, X)$ and $\varphi \in \mathscr{W} \mathscr{A} \mathscr{P}_{0}(\mathbb{R}, X)$, the subspace of $\mathscr{W} \mathscr{A} \mathscr{P}(\mathbb{R}, X)$ whose members have the zero function in the weak closure of the set of translates [3, Theorem 4.11]. The difficult part of answering the question is to handle the function $\varphi$. For this purpose we introduce a new generalization of almost periodic functions, which we call pseudo-almost periodic functions.

In this paper, we first set up some theorems of the indefinite integral of a pseudo-almost periodic function from $\mathbb{J}_{a}$ to $X$. Then we use these theorems to answer the question.

Received by the editors June 30, 1992 and, in revised form, August 20, 1992.

1991 Mathematics Subject Classification. Primary 43A60; Secondary 28B05.

Key words and phrases. Almost periodic functions, pseudo-almost periodic functions, integral. 
Definition 1. A subset $P$ of $\mathbb{J}_{a}$ is said to be relatively dense in $\mathbb{J}_{a}$ if there exists a number $l>0$ such that $[t, t+l] \cap P \neq \varnothing \quad\left(t \in \mathbb{J}_{a}\right)$.

A function $g \in \mathscr{C}(\mathbb{R}, X)$ is in $\mathscr{A} \mathscr{P}(\mathbb{R}, X)$ if and only if, for $\epsilon>0$, the set

$$
P(\epsilon)=\{\tau \in \mathbb{R}:\|g(t+\tau)-g(t)\|<\epsilon \text { for all } t \in \mathbb{R}\}
$$

is relatively dense in $\mathbb{R}[2$, Theorem 6.6]. A function $g \in \mathscr{A} \mathscr{P}(\mathbb{R}, X)$ is uniformly continuous.

Set

$$
\begin{aligned}
{\mathscr{P} \mathscr{A} \mathscr{P}_{0}}_{0}\left(\mathbb{J}_{a}, X\right)= & \left\{\varphi \in \mathscr{C}\left(\mathbb{J}_{a}, X\right): \lim _{t \rightarrow \infty} \frac{1}{t-a} \int_{a}^{t}\|\varphi(s)\| d s=0\right. \\
& \left.\left(\lim _{t \rightarrow \infty} \frac{1}{2 t} \int_{-t}^{t}\|\varphi(s)\| d s=0, \text { when } a=-\infty\right)\right\} .
\end{aligned}
$$

Definition 2. A function $f \in \mathscr{C}\left(\mathbb{J}_{a}, X\right)$ is called pseudo-almost periodic if

$$
f=\left.g\right|_{\mathbb{J}_{a}}+\varphi,
$$

where $g \in \mathscr{A} \mathscr{P}(\mathbb{R}, X)$ and $\varphi \in \mathscr{P} \mathscr{A} \mathscr{P}_{0}\left(\mathbb{J}_{a}, X\right)$. Denote by $\mathscr{P} \mathscr{A} \mathscr{P}\left(\mathbb{J}_{a}, X\right)$ all such functions. As in [7], $g$ and $\varphi$ are called the almost periodic component and the ergodic perturbation of $f$, respectively.

Definition 3. A closed subset $C$ of $\mathbb{J}_{a}$ is said to be an ergodic zero set in $\mathbb{J}_{a}$ if $m(C \cap[a, t]) /(t-a) \rightarrow 0$ as $t \rightarrow \infty \quad(m(C \cap[-t, t]) / 2 t \rightarrow 0$ as $t \rightarrow \infty$, when $a=-\infty)$.

The proof of the following proposition is straightforward.

Proposition 4. A function $\varphi \in \mathscr{C}\left(\mathbb{J}_{a}, X\right)$ is in $\mathscr{P}_{\mathscr{A}} \mathscr{P}_{0}\left(\mathbb{J}_{a}, X\right)$ if and only if, for $\epsilon>0$, the set $C_{\epsilon}=\left\{t \in \mathbb{J}_{a}:\|\varphi(t)\| \geq \epsilon\right\}$ is an ergodic zero subset in $\mathbb{J}_{a}$.

Proposition 5. Let $C$ be an ergodic zero set in $\mathbb{J}_{a}$. Then for any $\delta>0$ and $L>0$, there exists an interval $(u, v) \subset \mathbb{J}_{a}$ with the properties that $v-u>L$ and $m(C \cap(u, v))<\delta$.

Proof. If such a $(u, v)$ does not exist, one sees readily that

$$
\lim \inf _{t \rightarrow \infty} m(C \cap[a, t]) / t \geq \delta / 2 L
$$

$\left(\liminf \operatorname{in}_{t \rightarrow \infty} m(C \cap[-t, t]) / 2 t \geq \delta / 2 L\right.$, when $\left.a=-\infty\right)$.

Using Propositions 4 and 5, we can show that, for $g \in \mathscr{A} \mathscr{P}(\mathbb{R}, X)$, if $\left.g\right|_{\mathbf{J}_{a}} \in \mathscr{P} \mathscr{A} \mathscr{P}_{0}\left(\mathbb{J}_{a}, X\right)$, then $g=0$. In fact, since $g$ is uniformly continuous, for $\epsilon>0$ there is $\delta>0$ such that $\left\|g\left(t^{\prime}\right)-g\left(t^{\prime \prime}\right)\right\|<\epsilon$ whenever $t^{\prime}, t^{\prime \prime} \epsilon$ $\mathbb{R}$ with $\left|t^{\prime}-t^{\prime \prime}\right|<\delta$. Let $l>0$ be the number for $P(\epsilon)$ as in Definition

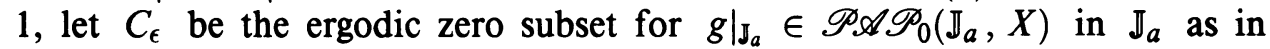
Proposition 4, and let $L=2 l$. By Proposition 5, for $\delta>0$ and $L>0$, there exists an interval $(u, v) \subset \mathbb{J}_{a}$ with the properties that $v-u>L$ and $m(C \cap(u, v))<\delta$. It follows that $\|g(t)\|<2 \epsilon, t \in(u, v)$. For $t \in \mathbb{R}$, since $v-u>2 l$, one can find $\tau \in P(\epsilon)$ such that $t+\tau \in(u, v)$. Therefore, $\|g(t)\| \leq\|g(t)-g(t+\tau)\|+\|g(t+\tau)\|<3 \epsilon$. Since $\epsilon>0$ is arbitrary, $g=0$. Thus

$$
\mathscr{P} \mathscr{A} \mathscr{P}\left(\mathbb{J}_{a}, X\right)=\left.\mathscr{A} \mathscr{P}(\mathbb{R}, X)\right|_{\mathbb{J}_{a}} \oplus \mathscr{P} \mathscr{A} \mathscr{P}_{0}\left(\mathbb{J}_{a}, X\right) .
$$

To show the next theorem, we need the following lemma. 
Lemma 6. Let $P$ be relatively dense in $\mathbb{J}_{a}$, and let $C$ be an ergodic zero set in $\mathbb{J}_{a}$. Then, for any given interval $[c, d] \subset \mathbb{R}$ and $\delta>0$, there exist $(u, v) \subset \mathbb{J}_{a}$ and $\tau \in P$ such that

$$
[c, d]+\tau \subset(u, v) \text { and } m(C \cap(u, v))<\delta .
$$

Proof. Let $l>0$ be the number for $P$ as in Definition 1 , and let $L=l+(d-c)$. By Proposition 5, there exists an interval $(u, v) \subset \mathbb{J}_{a}$ such that $m(C \cap(u, v))<$ $\delta$ and $L<v-u$. Since we can assume that $u-c \in \mathbb{J}_{a}$, we can choose $\tau \in[u-c, u-c+l] \cap P$. If $t \in[c, d]$,

$$
u<c+\tau \leq t+\tau \leq d+\tau \leq d+u-c+l<v
$$

that is, $[c, d]+\tau \subset(u, v)$.

Theorem 7. Let $a \in \mathbb{R}$, and let $f \in \mathscr{P} \mathscr{A} \mathscr{P}_{0}\left(\mathbb{J}_{a}, X\right)$. Define $F: \mathbb{J}_{a} \rightarrow X$ by $F(t)=\int_{a}^{t} f(u) d u$. Then $F \in \mathscr{P} \mathscr{A} \mathscr{P}\left(\mathbb{J}_{a}, X\right)$ if and only if there is a vector $A \in X$ such that $F-A \in \mathscr{P} \mathscr{A P}_{0}\left(\mathbb{J}_{a}, X\right)$.

Proof. The sufficiency is obvious. Now we show the necessity.

Since $F \in \mathscr{P A} \mathscr{P}\left(\mathbb{J}_{a}, X\right), F=\left.G\right|_{\mathbb{J}_{a}}+\Phi$, where $G \in \mathscr{A} \mathscr{P}(\mathbb{R}, X)$ and $\Phi \in \mathscr{P} \mathscr{A P}_{0}\left(\mathbb{J}_{a}, X\right)$. To show the necessity, we need to show that $G$ is a constant vector in $X$.

If it is not, there are $t^{\prime}, t^{\prime \prime} \in \mathbb{R}$ with $t^{\prime}<t^{\prime \prime}$ such that

$$
\left\|G\left(t^{\prime}\right)-G\left(t^{\prime \prime}\right)\right\|=\epsilon>0 .
$$

Since $G \in \mathscr{A} \mathscr{P}(\mathbb{R}, X)$, for any $\tau \in P(\epsilon / 4)$,

$$
\left\|G\left(t^{\prime}\right)-G\left(t^{\prime}+\tau\right)\right\|<\epsilon / 4 \text { and }\left\|G\left(t^{\prime \prime}\right)-G\left(t^{\prime \prime}+\tau\right)\right\|<\epsilon / 4
$$

Combining these three inequalities, we have

$$
\left\|G\left(t^{\prime}+\tau\right)-G\left(t^{\prime \prime}+\tau\right)\right\|>\epsilon / 2 \quad(\tau \in P(\epsilon / 4)) .
$$

$\Phi$ is uniformly continuous on $\mathbb{J}_{a}$ since $F$ and $G$ are. Let $\delta>0$ be such that $\|f\| \delta<\epsilon / 8$ and

$$
\left\|\Phi\left(t_{1}\right)-\Phi\left(t_{2}\right)\right\|<\epsilon / 16 \quad\left(t_{1}, t_{2} \in \mathbb{J}_{a},\left|t_{1}-t_{2}\right|<\delta\right) .
$$

Set

$$
\begin{aligned}
& C_{1}=\left\{t \in \mathbb{J}_{a}:\|\Phi(t)\| \geq \min \left\{\frac{\epsilon}{8\left(t^{\prime \prime}-t^{\prime}\right)}, \frac{\epsilon}{16}\right\}\right\}, \\
& C_{2}=\left\{t \in \mathbb{J}_{a}:\|f(t)\| \geq \min \left\{\frac{\epsilon}{8\left(t^{\prime \prime}-t^{\prime}\right)}, \frac{\epsilon}{16}\right\}\right\},
\end{aligned}
$$

and $C_{\epsilon}=C_{1} \cup C_{2}$. It follows from Proposition 4 that $C_{\epsilon}$ is an ergodic zero subset in $\mathbb{J}_{a}$. By Lemma 6 , there exist a $\tau_{0} \in P(\epsilon / 4)$ and $(u, v) \subset \mathbb{J}_{a}$ such that $\left[t^{\prime}, t^{\prime \prime}\right]+\tau_{0} \subset(u, v)$ and $m\left((u, v) \cap C_{\epsilon}\right)<\delta$.

We claim that $\left\|\Phi\left(t^{\prime}+\tau_{0}\right)\right\|<\epsilon / 8$ and $\left\|\Phi\left(t^{\prime \prime}+\tau_{0}\right)\right\|<\epsilon / 8$. In fact, if $t^{\prime}+\tau_{0} \in(u, v) \backslash C_{\epsilon}$, then $\left\|\Phi\left(t^{\prime}+\tau_{0}\right)\right\|<\epsilon / 16$; if $t^{\prime}+\tau_{0} \in(u, v) \cap C_{\epsilon}$, then by (2)

$$
\left\|\Phi\left(t^{\prime}+\tau_{0}\right)\right\| \leq\left\|\Phi\left(t^{\prime}+\tau_{0}\right)-\Phi(t)\right\|+\|\Phi(t)\|<\epsilon / 8,
$$

where $t \in(u, v) \backslash C_{\epsilon}$ is such that $\left|t-\left(t^{\prime}+\tau_{0}\right)\right|<\delta$.

Similarly we can show that $\Phi\left(t^{\prime \prime}+\tau_{0}\right)<\epsilon / 8$. 
Now

$$
\begin{aligned}
\| G\left(t^{\prime}\right. & \left.+\tau_{0}\right)-G\left(t^{\prime \prime}+\tau_{0}\right) \| \\
& \leq\left\|\int_{a}^{t^{\prime}+\tau_{0}}-\int_{a}^{t^{\prime \prime}+\tau_{0}} f(u) d u\right\|+\left\|\Phi\left(t^{\prime}+\tau_{0}\right)\right\|+\left\|\Phi\left(t^{\prime \prime}+\tau_{0}\right)\right\| \\
& <\left\|\int_{t^{\prime}+\tau_{0}}^{t^{\prime \prime}+\tau_{0}} f(u) d u\right\|+\frac{\epsilon}{4} \\
& =\int_{\left[t^{\prime}+\tau_{0}, t^{\prime \prime}+\tau_{0}\right] \backslash C_{\epsilon}}\|f(u)\| d u+\int_{\left[t^{\prime}+\tau_{0}, t^{\prime \prime}+\tau_{0}\right] \cap C_{\epsilon}}\|f(u)\| d u+\frac{\epsilon}{4} \\
& \leq\left|t^{\prime \prime}-t^{\prime}\right| \cdot \frac{\epsilon}{8\left(t^{\prime \prime}-t^{\prime}\right)}+\|f\| \delta+\frac{\epsilon}{4}<\frac{\epsilon}{2},
\end{aligned}
$$

which contradicts (1).

Denote by $\mathscr{C}_{0}\left(\mathbb{J}_{a}, X\right)$ the set of functions $f \in \mathscr{C}\left(\mathbb{J}_{a}, X\right)$ such that $f(t) \rightarrow 0$ as $t \rightarrow \infty$. A function $f \in \mathscr{C}\left(\mathbb{J}_{a}, X\right)$ is called asymptotically almost periodic if $f=\left.g\right|_{\mathrm{J}_{a}}+\varphi$, where $g \in \mathscr{A} \mathscr{P}(\mathbb{R}, X)$ and $\varphi \in \mathscr{C}_{0}\left(\mathbb{J}_{a}, X\right)$ [6]. Denote by $\mathscr{A} \mathscr{A} \mathscr{P}\left(\mathbb{J}_{a}, X\right)$ all such functions $f$. Since $\mathscr{C}_{0}\left(\mathbb{J}_{a}, X\right) \subset \mathscr{P} \mathscr{A} \mathscr{P}_{0}\left(\mathbb{J}_{a}, X\right)$, $\mathscr{A} \mathscr{A} \mathscr{P}\left(\mathbb{J}_{a}, X\right) \subset \mathscr{P} \mathscr{A} \mathscr{P}\left(\mathbb{J}_{a}, X\right)$. We have

Corollary $8[5,4.2]$. Let $a \in \mathbb{R}$, let $f \in \mathscr{C}_{0}\left(\mathbb{J}_{a}, X\right)$, and let $F: \mathbb{J}_{a} \rightarrow X$ be defined by $F(t)=\int_{a}^{t} f(u) d u$. Then $F \in \mathscr{A} \mathscr{A} \mathscr{P}\left(\mathbb{J}_{a}, X\right)$ if and only if $\lim _{t \rightarrow \infty} F(t)$ exists.

Proof. Necessity. Since $F \in \mathscr{A} \mathscr{A} \mathscr{P}\left(\mathbb{J}_{a}, X\right), F=\left.G\right|_{\mathbf{J}_{a}}+\Phi^{\prime}$, where $G \in$ $\mathscr{A} \mathscr{P}(\mathbb{R}, X)$ and $\Phi^{\prime} \in \mathscr{C}_{0}\left(\mathbb{J}_{a}, X\right)$. By Theorem $7, F=A+\Phi$, where $A \in X$ and $\Phi \in \mathscr{P} \mathscr{A} \mathscr{P}_{0}\left(\mathbb{J}_{a}, X\right)$. The uniqueness of the decomposition implies that $G=A$ and $\Phi \in \mathscr{C}_{0}\left(\mathbb{J}_{a}, X\right)$. Therefore, $F(t)=\int_{a}^{t} f(u) d u \rightarrow A$ as $t \rightarrow \infty$.

The sufficiency is easy to prove.

The following example of $[5,4.1]$ can also be used here to show that the bounded integral of a pseudo-almost periodic function may fail to be pseudoalmost periodic even in the numerical case.

Example 9. Consider the function $f: \mathbb{J}_{1} \rightarrow \mathbb{R}$ defined by

$$
f(t)=\left(\frac{1}{t}\right) \cos (\log t) \quad\left(t \in \mathbb{J}_{1}\right) .
$$

Since $f(t) \rightarrow 0$ as $t \rightarrow \infty, f(t) \in \mathscr{P} \mathscr{A} \mathscr{P}_{0}\left(\mathbb{J}_{1}\right)$. The corresponding indefinite integral

$$
F(t)=\int_{1}^{t} f(u) d u=\sin (\log t) \quad\left(t \in \mathbb{J}_{1}\right)
$$

defines a bounded function on $\mathbb{J}_{1}$; however, $F \notin \mathscr{P} \mathscr{A} \mathscr{P}\left(\mathbb{J}_{a}\right)$. That is because

$$
\frac{1}{r} \int_{1}^{r}|\sin (\log t)| d t \nrightarrow 0
$$

as $r \rightarrow \infty$ and neither does $\frac{1}{r} \int_{1}^{r}|\sin (\log t)-A| d t$ for any $A \in \mathbb{C}$. But, if $F \in \mathscr{P} \mathscr{A} \mathscr{P}\left(\mathbb{J}_{a}\right), F$ differs from some member of $\mathscr{P} \mathscr{A} \mathscr{P}_{0}\left(\mathbb{J}_{a}\right)$ by a constant (Theorem 7).

Let $f \in \mathscr{P} \mathscr{A} \mathscr{P}\left(\mathbb{J}_{a}, X\right)$, and define $F(t)=\int_{a}^{t} f(u) d u$ for $t \in \mathbb{J}_{a}$. To show $F \in \mathscr{P} \mathscr{A} \mathscr{P}\left(\mathbb{J}_{a}, X\right)$, we need to treat two cases. Theorem 7 allows us to control 
the ergodic perturbation of $f$. We utilize the work of Kadets [4] in treating the almost periodic part of $f$. Also, as in [5], we need the following lemmas. In the lemmas, for a subset $B$ of $X, \overline{\operatorname{cco}}(B)$ denotes the closed convex circled hull of $B$.

Lemma 10. Let $a \in \mathbb{R}$, and let $f \in \mathscr{P} \mathscr{A P}\left(\mathbb{J}_{a}, X\right)$. Let $g$ and $\varphi$ be the almost periodic component and the ergodic perturbation of $f$, respectively. If $F(t)=\int_{a}^{t} f(u) d u$ and $G_{a}(t)=\int_{a}^{t} g(u) d u$ for $t \in \mathbb{J}_{a}$, then

$$
G_{a}\left(\mathbb{J}_{a}\right) \subset 2 \overline{\mathrm{cco}}\left(F\left(\mathbb{J}_{a}\right)\right) \text {. }
$$

Proof. Suppose that the conclusion does not hold; we can find a $t_{0} \in \mathbb{J}_{a}$ such that $G_{a}\left(t_{0}\right) \notin 2 \overline{\operatorname{cco}}\left(F\left(\mathbb{J}_{a}\right)\right)$. In this case, we point out that $\|\varphi\| \neq 0$; otherwise we will have the conclusion. Also, $t_{0} \neq a$ because $G_{a}(a)=0 \in 2 \overline{\operatorname{cco}} F\left(\mathbb{J}_{a}\right)$. By the Hahn-Banach theorem, there is an $x^{*} \in X^{*}$ such that

$$
\min _{y \in 2 \operatorname{coc}\left(F\left(J_{a}\right)\right)}\left|x^{*}\left(G_{a}\left(t_{0}\right)-y\right)\right|=\epsilon>0 \text {. }
$$

Let

$$
0<\delta=\min \left\{\frac{\epsilon}{3\left\|x^{*}\right\|\left(t_{0}-a\right)} ; \frac{\epsilon}{3\left\|x^{*}\right\|\|\varphi\|}\right\}
$$

and

$$
C=\left\{t \in \mathbb{J}_{a}:\|\varphi(t)\| \geq \delta\right\} .
$$

By Proposition 4, $C$ is an ergodic zero subset of $\mathbb{J}_{a}$. For $\delta>0$ and $g \in$ $\mathscr{A} \mathscr{P}(\mathbb{R}, X)$, let $P(\delta)$ be the relatively dense subset of $\mathbb{R}$. By Lemma 6 , there are $(u, v) \subset \mathbb{J}_{a}$ and a $\tau \in P(\delta)$ such that $\left[a, t_{0}\right]+\tau \subset(u, v)$ and $m((u, v) \cap$ $C)<\delta$. Now,

$$
\begin{aligned}
\epsilon & =\min _{y \in 2 \cos \left(F\left(\mathbf{J}_{a}\right)\right)}\left|x^{*}\left(G_{a}\left(t_{0}\right)-y\right)\right| \\
\leq & \left|x^{*}\left\{G_{a}\left(t_{0}\right)-\left[F\left(t_{0}+\tau\right)-F(a+\tau)\right]\right\}\right| \\
\leq & \left|x^{*}\left\{G_{a}\left(t_{0}\right)-\int_{a}^{t_{0}} g(u+\tau) d u\right\}\right| \\
& +\left|x^{*}\left\{\int_{a}^{t_{0}} g(u+\tau) d u-\int_{a+\tau}^{t_{0}+\tau} f(u) d u\right\}\right| \\
\leq & \left\|x^{*}\right\| \int_{a}^{t_{0}}\|g(u)-g(u+\tau)\| d u+\left|x^{*}\left\{\int_{a}^{t_{0}} \varphi(u+\tau) d u\right\}\right| \\
\leq & \left\|x^{*}\right\|\left(t_{0}-a\right) \delta+\int_{a}^{t_{0}}\left|x^{*}\{\varphi(u+\tau)\}\right| d u \\
= & \left\|x^{*}\right\|\left(t_{0}-a\right) \delta+\int_{\left[a+\tau, t_{0}+\tau\right] \backslash C}\left|x^{*}\{\varphi(u)\}\right| d u+\int_{\left[a+\tau, t_{0}+\tau\right] \cap C}\left|x^{*}\{\varphi(u)\}\right| d u \\
< & \left\|x^{*}\right\|\left(t_{0}-a\right) \delta+\delta\left\|x^{*}\right\|\left(t_{0}-a\right)+\delta\|\varphi\|\left\|x^{*}\right\|<\epsilon,
\end{aligned}
$$

a contradiction.

Lemma $11[5,4.5]$. Let $a \in \mathbb{R}$, and let $g \in \mathscr{A} \mathscr{P}(\mathbb{R}, X)$. Put $G_{a}(t)=\int_{a}^{t} g(u) d u$ for $t \in \mathbb{J}_{a}$, and set

$$
G(t)= \begin{cases}\int_{0}^{t} g(u) d u, & t \in \mathbb{J}_{0}, \\ -\int_{t}^{0} g(u) d u, & t \in \mathbb{R} \backslash \mathbb{J}_{0} .\end{cases}
$$


Then

$$
G(\mathbb{R}) \subset G([0,|a|])+2 \overline{\operatorname{cco}}\left(G_{a}\left(\mathbb{J}_{a}\right)\right) .
$$

Taken together, Lemmas 10 and 11 yield the following result.

Lemma 12. Let $a, f, g, \varphi, F$, and $G_{a}$ be as in Lemma 10. If $F\left(\mathbb{J}_{a}\right)$ is bounded [weakly relatively compact] \{relatively compact\} in $X$, then the same is true for $G(\mathbb{R})$.

Let $\mathscr{B}\left(\mathbb{J}_{a}, X\right)$ denote the set of all bounded functions from $\mathbb{J}_{a}$ to $X$. Now we have the following theorem.

Theorem 13. Let $a, f, g, \varphi$, and $F$ be as in Lemma 10. Suppose either

(i) $F \in \mathscr{B}\left(\mathbb{J}_{a}, X\right)$ and $X$ does not contain an isomorphic copy of $c_{0}$, or

(ii) $F\left(\mathbb{J}_{a}\right)$ is weakly relatively compact in $X$.

Then $F \in \mathscr{P} \mathscr{A} \mathscr{P}\left(\mathbb{J}_{a}, X\right)$ if and only if there is an $A \in X$ such that $\Phi$, defined by

is in $\mathscr{P} \mathscr{A} \mathscr{P}_{0}\left(\mathbb{J}_{a}, X\right)$.

$$
\Phi(x)=\int_{0}^{x} \varphi(u) d u-A,
$$

Proof. Necessity. We define the indefinite integrals $G_{a}: \mathbb{J}_{a} \rightarrow X$ and $G: \mathbb{R} \rightarrow X$ of $g$ as in Lemma 11.

Since (i) or (ii) is satisfied, Lemma 12 shows that at least one of the following holds: $c_{0} \not \subset X, G(\mathbb{R})$ is bounded, and $G(\mathbb{R})$ is weakly relatively compact in $X$. By [4, Theorem 1] in case (i), or by [4, Theorem 2] in case (ii), $G$ is almost periodic. Therefore, $G_{a}=\left.G\right|_{J_{a}}-G(a) \in \mathscr{P} \mathscr{A} \mathscr{P}\left(\mathbb{J}_{a}, X\right)$, as is $\psi$, where

$$
\psi(t)=\int_{a}^{t} \varphi(u) d u=F(t)-\int_{a}^{t} g(u) d u .
$$

Now the necessity follows from Theorem 7.

The sufficiency is easy to prove; we omit the proof.

The following theorem was shown in [5, Theorem 4.11].

Theorem 14. Let $f=g+\varphi$, where $g \in \mathscr{A} \mathscr{P}(\mathbb{R}, X)$ and $\varphi \in \mathscr{C}_{0}(\mathbb{R}, X)$. Define $F: \mathbb{R} \rightarrow X$ by $F(t)=\int_{0}^{t} f(u) d u$. Then $F$ is in $\mathscr{W} \mathscr{P}(\mathbb{R}, X)$ if and only if either

(i) $F(\mathbb{R})$ is weakly relatively compact in $X$ or

(ii) $c_{0} \not \subset X$ and $F \in \mathscr{B}(\mathbb{R}, X)$,

and the following limits exist and satisfy

$$
\lim _{t \rightarrow \infty} \int_{0}^{t} \varphi(u) d u=\lim _{t \rightarrow-\infty} \int_{0}^{t} \varphi(u) d u .
$$

Since $\mathscr{C}_{0}(\mathbb{R}, X) \subsetneq \mathscr{W} \mathscr{A} \mathscr{P}_{0}(\mathbb{R}, X)$, Ruess and Summers pointed out in [5, p. 33] that Theorem 14 does not answer the question: when is the integral of an $f \in \mathscr{W} \mathscr{A} \mathscr{P}(\mathbb{R}, X)$ again in $\mathscr{W} \mathscr{A} \mathscr{P}(\mathbb{R}, X)$ ?

The next theorem answers this question. Before stating the theorem, we show that if $f \in \mathscr{W} \mathscr{A} \mathscr{P}(\mathbb{R}, X)$ and $x^{*} \in X^{*}$, then $\left.x^{*} f\right|_{\mathbf{J}_{0}} \in \mathscr{P} \mathscr{A} \mathscr{P}\left(\mathbb{J}_{0}\right)$, where $\mathbb{J}_{0}=$ $[0, \infty)$. In fact, $f=g+\varphi$, where $g \in \mathscr{A} \mathscr{P}(\mathbb{R}, X)$ and $\varphi \in \mathscr{W} \mathscr{A} \mathscr{P}_{0}(\mathbb{R}, X)[3$, Theorem 4.11]. $x^{*} g \in \mathscr{A} \mathscr{P}(\mathbb{R})$, and $x^{*} \varphi \in \mathscr{W} \mathscr{A} \mathscr{P}_{0}(\mathbb{R})$. Since $\mathscr{W} \mathscr{A} \mathscr{P}_{0}(\mathbb{R}) \subset$ $\mathscr{P} \mathscr{A} \mathscr{P}_{0}(\mathbb{R})[1,4.3 .13], x^{*} \varphi \in \mathscr{P} \mathscr{A} \mathscr{P}_{0}(\mathbb{R})$. So $\left.x^{*} \varphi\right|_{\mathrm{J}_{0}} \in \mathscr{P} \mathscr{A} \mathscr{P}_{0}\left(\mathbb{J}_{0}\right)$ and $\left.x^{*} f\right|_{\mathbf{J}_{0}} \in$ $\mathscr{P} \mathscr{A} \mathscr{P}\left(\mathbb{J}_{0}\right)$. 
Theorem 15. Let $f \in \mathscr{W} \mathscr{A} \mathscr{P}(\mathbb{R}, X)$. Define $F: \mathbb{R} \rightarrow X$ by $F(t)=\int_{0}^{t} f(u) d u$. Then $F$ is in $\mathscr{W} \mathscr{A}(\mathbb{R}, X)$ if and only if either

(i) $F(\mathbb{R})$ is weakly relatively compact in $X$, or

(ii) $c_{0} \not \subset X$ and $F \in \mathscr{B}(\mathbb{R}, X)$,

and there is a vector $A \in X$ such that

$$
\psi-A \in \mathscr{W} \mathscr{A} \mathscr{P}_{0}(\mathbb{R}, X),
$$

where $\psi: \mathbb{R} \rightarrow X$ is defined by $\psi(t)=\int_{0}^{t} \varphi(u) d u$.

Proof. Set $G(t)=\int_{0}^{t} g(u) d u$ for $t \in \mathbb{R}$. With a proof similar to that for Lemma 12 , we can show that if $F(\mathbb{R})$ is bounded (weakly relatively compact) in $X$, then the same is true for $G(\mathbb{R})$.

Necessity. Since $F \in \mathscr{W} \mathscr{A} \mathscr{P}(\mathbb{R}, X), F(\mathbb{R})$ is weakly relatively compact, as is $G(\mathbb{R})$. So $G \in \mathscr{A} \mathscr{P}(\mathbb{R}, X)$ [4, Theorem 2].

Now we show that (4) holds for some $A \in X$. Since $\psi \in \mathscr{W} \mathscr{A} \mathscr{P}(\mathbb{R}, X)$,

$$
\psi=G_{1}+\Phi_{1},
$$

where $G_{1} \in \mathscr{A} \mathscr{P}(\mathbb{R}, X)$ and $\Phi_{1} \in \mathscr{W} \mathscr{A} \mathscr{P}_{0}(\mathbb{R}, X)$. We claim that $G_{1}$ is a constant function. For, suppose that there are $t_{1}, t_{2} \in \mathbb{R}$ such that $G_{1}\left(t_{1}\right) \neq$ $G_{1}\left(t_{2}\right)$. The almost periodicity of $G_{1}$ makes it possible to assume that $t_{1}$ and $t_{2}$ are in $\mathbb{J}_{0}$. Then we can find a $x^{*} \in X^{*}$ such that $x^{*} G_{1}\left(t_{1}\right) \neq x^{*} G_{1}\left(t_{2}\right)$. Since $\psi \in \mathscr{W} \mathscr{A} \mathscr{P}(\mathbb{R}, X),\left.x^{*} \psi\right|_{\mathrm{J}_{0}} \in \mathscr{P} \mathscr{A} \mathscr{P}\left(\mathbb{J}_{0}\right)$. By Theorem 7 there are an $A_{x^{*}} \in \mathbb{C}$ and a $\Phi_{x^{*}} \in \mathscr{P} \mathscr{A} \mathscr{P}_{0}\left(\mathbb{J}_{0}\right)$ such that

$$
x^{*} \psi(t)=\int_{0}^{t} x^{*} \varphi(u) d u=A_{x^{*}}+\Phi_{x^{*}}(t) \quad\left(t \in \mathbb{J}_{0}\right) .
$$

Comparing (6) with (5) and using the uniqueness of the decomposition, we conclude that $x^{*} G_{1}$ is a constant function, a contradiction.

Sufficiency. It is obvious that $\psi \in \mathscr{W} \mathscr{A} \mathscr{P}(\mathbb{R}, X)$ if (4) holds for some $A \in$ $X$. By the first paragraph in the proof and [4, Theorems 1 and 2], either (i) or (ii) is a sufficient condition for $G$ to be in $\mathscr{A} \mathscr{P}(\mathbb{R}, X)$, so $F \in \mathscr{W} \mathscr{A} \mathscr{P}(\mathbb{R}, X)$.

Remark 16. Theorem 14 is a corollary of Theorem 15. In fact, in Theorem 14, $A=\lim _{t \rightarrow \infty} \int_{0}^{t} \varphi(u) d u$.

\section{ACKNOWLEDGMENT}

The contents of this paper is a part of the author's Ph.D. work under the supervision of Professor P. Milnes at the University of Western Ontario. The author would like to acknowledge his indebtedness to his supervisor, Professor $P$. Milnes, for advice and encouragement given while this paper was being written.

\section{BIBLIOGRAPHY}

1. J. F. Berglund, H. D. Junghenn, and P. Milnes, Analysis on semigroups: Function spaces, compactifications, representations, Wiley, New York, 1989.

2. C. Corduneanu, Almost periodic functions, Chelsea, New York, 1989.

3. K. Deleeuw and I. Glicksberg, Applications of almost periodic compactifications, Acta Math. 105 (1961), 63-97. 
4. M. I. Kadets, On the integration of almost periodic functions with values in a Banach space, Funct. Anal. Appl. 3 (1969), 228-230.

5. W. M. Ruess and W. H. Summers, Integration of asymptotically almost periodic functions and weak asymptotic almost periodicity, Dissertationes Math. (Rozprawy Math.) 279 (1989).

6. S. Zaidman, Almost-periodic functions in abstract spaces, Pitman, London, 1985.

7. C. Zhang, Pseudo almost periodic functions and their applications, thesis, University of Western Ontario, 1992.

Department of Mathematics, The University of British Columbia, Vancouver, CANADA V6T 122

E-mail address: czhang@math.ubc.ca 